\title{
Application of Particle Swarm Optimization in High-precision Fundamental Matrix Estimation
}

\author{
Yanju Liu, Zedong Li* Hongwei Gao* \\ School of Information Science \& Engineering Shenyang Ligong University, \\ Shenyang, 110159, China \\ *Corresponding authors' Email: lizedong2008@163.com,ghw1978@sohu.com
}

\begin{abstract}
This paper discusses the fundamental matrix that is recovered by the use of the characteristic points of the images collected by camera. Firstly, the corners are detected by Harris Operator, but there are error matching points in the set of characteristic points. Secondly, the relaxation matching is used to search fine matching point pairs. Thirdly, in order to gain better points, the objective function is founded by interrelated geometry theory. Lastly, the fundamental matrix will be solved with corresponding matching points best matching points are gained. The PSO algorithm is used to optimize the fundamental matrix. And the epipolar line is recovered by the fundamental matrix. The results of experiments show that better effects are obtained.
\end{abstract}

Keywords: Harris Operator; relaxation matching; fundamental matrix; PSO

\section{Introduction}

The same object from different location of image matching is one of the important methods to obtain three-dimensional information of the object located in the three-dimensional space. To match images correctly via camera will help a true reflection of objects in the spatial information. As the MIT professor Marr established the basic framework of computer vision, since the 1990s, Hartley, Long QUAN, Philip Torr, Sami Brand and others have done a lot of work in the accurate estimation of fundamental matrix. Myron Z. Brown, etc. summed up the development of the three-dimensional matching technology during 1993 to 2003, and focused on block processing and realtime systems. In 2002, Scharstein and Szeliski divided the visual process into the three-dimensional visual module-calculation of cost function, accumulation of cost function, parallax calculation or optimization and parallax refinement. Since then, the development of stereo matching goes into systematic and rapid development. Feature point matching can be divided into three steps: firstly, extract a set of feature points in matching images, secondly, find the corresponding relationship in two or more sets of characteristic points; finally, according to the set of corresponding relationship, select matching feature points. Under normal circumstances, due to the noise, real feature point is lost, or the pseudo feature point occurs, which can affect the matching results. Therefore, an objective function need to be constructed, which can eliminate false feature points, extract exactly, optimize calculation results and establish the optimal fundamental matrix. In this paper, firstly, fundamental matrix that is obtained by feature points is optimized by PSO algorithm; secondly, restore the epipolar line of corresponding matching points in the image; at last, compare the recovery effects of epipolar before and after using the PSO algorithm. 


\section{Characteristic points extraction}

In 1988, Harris and Stephens presented an operator based on feature extraction of still images named Harris Operator. According to the definition of Harris Operator, the point whose horizontal and vertical curvature is higher than others" which are around the point calculated in the domain of local neighborhood is the feature point.

Harris Operator has three steps. The first is that the correlation matrix of every pixel is necessarily calculated. We make convolution between Gaussian function and correlation matrix. This can reduce noise effect.

$$
C(i, j)=G(s) \bigotimes M
$$

The matrix $M$ of the formula (1) is correlation matrix, which is expected.

$$
M=\left[\begin{array}{cc}
g_{x}^{2} & g_{x} g_{y} \\
g_{x} g_{y} & g_{y}^{2}
\end{array}\right]
$$

where $M$ is the Harris corner operator; $g_{x}$ is the $x$ direction of the gradient; $g_{y}$ is the $y$ direction of the gradient and $G(s)$ is the Gaussian template.

The second is that the response of the corner point of the image will be calculated.

$$
I=\operatorname{det}(c)-K \times t r^{2}(C)
$$

$I$ is curvature value of every point of the images, det is the determinant of the matrix $c, t r$ is the trace of the matrix $c, K$ is an experience value.

The third is that the maximum module points will be searched in bound of $w * w$. Here, $w * w$ is the scale of the image collected by us. If the response of the corner is greater the threshold we set previously, this corner will be considered as corner that is necessary for us.

\section{Fundamental matrix estimation}

\subsection{Epipolar line equation}

Epipolar line is an intersection line that is determined by two geometrical plans, one being determined by camera optical center and space objects, the other being photographic plane of camera. Therefore, epipolar line equation passes the corresponding match points of the reference image and the matching image. So, the epipolar is very useful to fix the corresponding matching points. Meanwhile, the epipolar equation is also an important equation to obtain a fundamental matrix.
The epipolar mathematical equation is made up of fundamental matrix and corresponding matching points.

$$
u_{2}^{T} F u_{1}=0
$$

where $F$ is the question for the matrix; $u_{1}$ and $u_{2}$ is the feature point of reference image and matching image extracted by Harris Operator.

If the value of equation is zero, it explains that the corresponding matching points locate the identical straight line. So the fundamental matrix is very important. The precision of fundamental matrix will influence the results of epipolar rectification.

\subsection{Relaxation matching}

According to Harris Operator, we can gain lots of characteristic points and there are many redundancy characteristic points. And the redundancy points will be dispelled. So the relaxation algorithm is used to get rid of false matching points.

The relaxation matching has two sections primarily. Firstly, coarse matching is used by gray statistical distribution next to the characteristic point. And the standard of partial match will be founded by normalized correlation. The characteristic points detected by Harris Operator will be divided into many-to-many pairs.

For example, there is characteristic point named $m_{1}$ in the reference image. The self-correlation window will be defined. The window takes $m_{1}\left(u_{1}, v_{1}\right)$ as center and the scale is $(2 n+1) \times(2 m+1)$. Then, the characteristic point will be searched by the scale of $(2 d u+1) \times(2 d v+1)$ in the matching image. If the characteristic point named $m_{2}\left(u_{2}, v_{2}\right)$ is found, selfcorrelation coefficient between $m_{1}$ and $m_{2}$ will be calculated.

$$
\begin{aligned}
& \text { Score }\left(m_{1}, m_{2}\right)= \\
& \frac{\sum_{i=-n}{ }^{n} \sum_{i=-m}{ }^{m}\left[I_{1}\left(u_{1}+i, v_{1}+j\right)-\overline{I_{1}\left(u_{1}, v_{1}\right)}\right]}{(2 n+1)(2 m+1)} \times \\
& \frac{\sum_{i=-n}{ }^{n} \sum_{i=-m}{ }^{m}\left[I_{2}\left(u_{2}+i, v_{2}+j\right)-\overline{I_{2}\left(u_{2}, v_{2}\right)}\right]}{\sqrt{\sigma^{2}\left(I_{1}\right) \times \sigma^{2}\left(I_{2}\right)}}
\end{aligned}
$$

where $\overline{I_{k}(u, v)}=\frac{\sum_{i=-n}{ }^{n} \sum_{i=-m}{ }^{m} I_{k}(u+i, v+j)}{(2 n+1)(2 m+1)} \overline{I_{k}}$ is gray mean of the point $(u, v), \sigma\left(I_{k}\right)$ is defined standard deviation of neighbour of $(2 n+1) \times(2 m+1), \sigma\left(I_{k}\right)$ is defined, as follows:

$$
\sigma\left(I_{k}\right)=\sqrt{\frac{\sum_{i=-n}{ }^{n} \sum_{i=-m}{ }^{m}\left[I_{k}(u, v)-\overline{I_{k}(u, v)}\right]}{(2 n+1)(2 m+1)}}
$$

We will set a threshold for the self-correlation coefficient. If the value of $\sigma\left(I_{k}\right)$ is greater than the threshold we set, the characteristic point will be reserved. 
And we have to set the scale of correlation window and $(d u, d v)$.

The candidate matching points of coarse matching can be gained by threshold processing, but these matching points may have some problems. For example, the characteristic point of the reference image will match few characteristic points of the matching image.

How do we resolve the problem? Secondly, we are going to solve ambiguous problems of coarse matching for fine matching. The fine matching has four steps.

The first step is that matching intensities of each candidate matching points should be calculated.

The second step is that the transparency of each matching characteristic point will be calculated. As follows:

$$
U_{A}=1-\frac{S_{M}^{(1)}}{S_{M}^{(2)}}
$$

where $S_{M}^{(1)}$ is the maximum matching intensity of point $X$, and $S_{M}{ }^{(2)}$ is the sub-maximum matching intensity of point $X$, then the value of result $U_{A}$ changes between 1 (clear) and 0 (fuzzy).

The third step is that the matching intensity and clarity of all candidate matching pairs are kept in the table $T_{S M}$ and $T_{U A}$. They are sorted in descending order, and the top 60 percent of table are cut out. If the matching intensity and clarity of one candidate matching pair are both kept in the top 60 percent of table $T_{S M}$ and $T_{U A}$, the candidate matching pair is regarded as the true matching pair. If the two feature points of one candidate matching pair don't correspond to the other point about maximum matching intensity, the candidate matching pair is regarded as the false matching pair. After that, the true matching pair and the false matching pair are both eliminated from candidate matching pairs.

The forth step is to return to the first step and process the next iteration.are not any true matching pairs. And all of the true matching pairs are used to compute transformation matrix.

\subsection{Objective function}

The Relaxation matching has been used after Harris Operator; the fine characteristic points have been grained. And, the fine fundamental matrix is our target. So the fine matching points is extracted. The objective function is found.

The objective function is a function of interested target (a variable) and related factors (some variables), that is, a question of function relationship is solved according to the known conditions which are thought have been set. In geometry, if the point is located in the straight line, the distance between the point and straight line is zero. In this paper, according to the theory, the object function is found.

In addition, according to the definition of the objective function and theory, the epipolar equation of characteristic points of the reference image has the same location in matching image. If the corresponding matching point locates the straight line, which is the epipolar equation, the distance is zero. Namely, epipolar equation of any characteristic point in reference image will pass extremely corresponding point in matching image.

Thus, the objective function named $d$ is set as follows:

$$
d=\frac{\left|u_{i}^{T} F u_{j}\right|}{\sqrt{{a_{i}{ }^{2}+b_{j}^{2}}^{2}}}
$$

where $\left(u_{i} \& u_{j}\right)$ is the characteristic point extracted from the matching image; $F$ is a fundamental matrix of the two cameras and $\left(a_{i}, b_{j}\right)$ is the coefficient of epipolar equation of the characteristic point in matching image. If the characteristic points of reference image and feature points of matching image can match each other to satisfy the above equations, then the distance between the matching point and the polar is zero.

\subsection{Fundamental matrix calculated}

In the stereo vision system, because of the differences in the camera spatial location, the locations of space objects is variable in projection plane. The fundamental matrix can reflect relationship of projection matrix between two cameras. Therefore, the fundamental matrix that is determined is a great significance for three-dimensional reconstruction.

The fundamental matrix is necessary to be solved well because it plays a determining role in the field of computer vision. So, we have finished many tasks to solve the problem of fundamental matrix. For example, the characteristic points were extracted.

According to the epipolar line constraints, feature matching points of the two images are collinear.

$$
\left[\begin{array}{lll}
u_{i} & v_{i} & 1
\end{array}\right]\left[\begin{array}{lll}
F_{11} & F_{12} & F_{13} \\
F_{21} & F_{22} & F_{23} \\
F_{31} & F_{32} & F_{33}
\end{array}\right]\left[\begin{array}{c}
u_{j} \\
v_{j} \\
1
\end{array}\right]=0
$$

In equation (9), matrix $F$ is the to be solved fundamental matrix, in which $F_{33}$ is a known quantity. We consider that the elements of the matrix $F$ include the same constant, so the elements of matrix are $F$ divided by $F_{33}$. The value of $F_{33}$ is one. So we only need 
eight groups of matching points and we can also decrease the error of calculation. $\left(u_{i}, v_{i}\right)$ and $\left(u_{j}, v_{j}\right)$ are corresponding matching points between matching image and reference image.

The formula (9) is simplified set of equations, as follows:

$$
\left\{\begin{array}{l}
u_{1}{ }^{1} u_{2}{ }^{1} F_{11}+v_{1}{ }^{1} u_{2}{ }^{1} F_{21}+u_{2}{ }^{1} F_{31}+u_{1}{ }^{1} v_{2}{ }^{1} F_{12}+ \\
v_{1}{ }^{1} v_{2}{ }^{1} F_{22}+v_{2}{ }^{1} F_{32}+u_{1}{ }^{1} F_{13}+v_{1}{ }^{1} F_{23}=-1 \\
u_{1}{ }^{2} u_{2}{ }^{2} F_{11}+v_{1}{ }^{2} u_{2}{ }^{2} F_{21}+u_{2}{ }^{2} F_{31}+u_{1}{ }^{2} v_{2}{ }^{2} F_{12}+ \\
v_{1}{ }^{2} v_{2}{ }^{2} F_{22}+v_{2}{ }^{2} F_{32}+u_{1}{ }^{2} F_{13}+v_{1}{ }^{2} F_{23}=-1 \\
\ldots
\end{array}\right.
$$

As shown in equations, $F_{11}-F_{32}$ are eight unknown variables of the fundamental matrix. At least, the eight groups of matching points are extracted in the reference images and matching images. In order to improve the calculation accuracy of the fundamental matrix, matching point is greater than the eight pairs and the least-squares will be operated for operation of given data.

\section{PSO optimization algorithm}

Originated from behavioral research of prey of birds, Dr. Eberhart and Dr. kennedy invented a kind of optimization algorithm, which is Particle Swarm Optimization (PSO) algorithm. PSO algorithm is a kind of intelligent computation technology which is based on group method. The PSO algorithm is mainly used for the global optimal solution. The PSO is an initialized group of random particles (random solutions). In each iteration, the particles renew itself by tracking the two "extremum", one being the particle itself to find the best solution called individual extremum point (its position indicated by pbest), the other extreme point of the global PSO being found in the entire population at present (its position indicated by gbest ). After finding the two best solutions, according to the equation (10) and (11), the velocity and position of particle is updated.

$$
\begin{gathered}
v_{i d}{ }^{k+1}=v_{i d}{ }^{k}+c_{1} \text { rand }_{1}{ }^{k}\left(\text { pbest }_{i d}{ }^{k}-x_{i d}{ }^{k}\right) \\
+c_{2} \text { rand }_{2}{ }^{k}\left(\text { gbest }_{i d}{ }^{k}-x_{i d}{ }^{k}\right) \\
x_{i d}{ }^{k+1}=x_{i d}{ }^{k}+v_{i d}{ }^{k+1}
\end{gathered}
$$

where $v_{i d}{ }^{k}$ is the speed of dimension in $k$ second iteration of particle $i ; c_{1}$ and $c_{2}$ are the factors of acceleration (called learning factor) and adjust the maximum step size of fighting direction of particle in global account and part; rand $_{1,2}$ is a random number during $[0,1] ; x_{i d}{ }^{k}$ is $d$-dimensional current position of particle $i$ in $k$ second iteration; pbest ${ }_{i d}$ is the particle $i$ position of individual extreme points in $d$-dimensional (coordinates); gbest $_{\text {id }}$ is global extreme position of a whole population in $d$-dimensional location. The parameters are optimized based on PSO, as follows:

Step 1: Population is initialized randomly. The location and speed of initial point is usually randomly generated in adjacent space. The initial particle number is 100 and $d=200$. The individual extremum is calculated respectively, and the best individual extremum is the global extremum.

Step 2: Each particle of population is evaluated. If the individual extremum is better than the current, the individual extremum will be updated. If the best extremum of all individual extremum is better than the current, the global extremum will be updated.

Step 3: The population is updated according to equation (10) and (11). If the terminative conditions are met, then stop; otherwise, switch to step 2.

Because the fundamental matrix is important in searching the matching feature points of images gathered by camera, we should gain a precise matrix. It reflects a kind of relationship between sequential images. Using the PSO algorithm, we can optimize the data, which are the elements of the fundamental matrix. The data optimized by PSO algorithm is accurate to match the feature points. The results that we optimize the fundamental matrix will be expressed as follows.

\section{Analysis of experimental results}

\subsection{Feature points extracted based on harris op- erator}

Seeing from the figures captured by camera, feature points which are extracted by Harris Operator locate in various regions of the images and reflect the characteristics of images.

\subsection{The polar of corresponding characteristic po- ints restored by fundamental matrix calcu- lated by least squares}

The epipolar line above the figures is restored by fundamental matrix that is calculated by least squares. According to the epipolar line in the figures, the fundamental matrix that is calculated by feature points is directly extracted. According to the figure, the errors of matching epipolar exist. In order to improve the precision of epipolar, we need to eliminate false matching points. We will found an object function which can get rid of the false matched points. If the matched points locate collinear, the value of distance between point and straight line which is corresponding is zero. According to the principle, we found the 

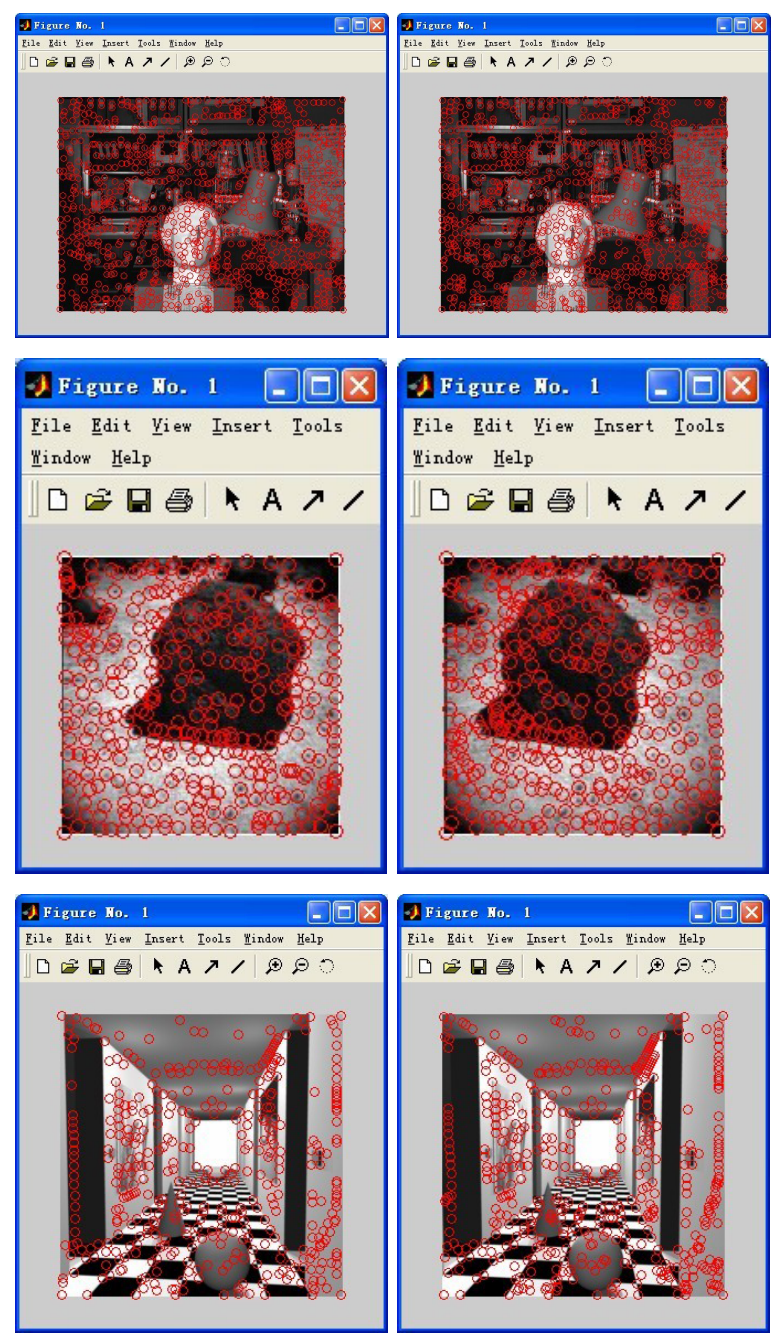

Figure 1 Detection result of harris operator

object function and we will set a threshold. If the value of distance is less than the threshold, we can consider corresponding point that is matching point. If the number of point whose distance is greater than the threshold, we will consider the point whose distance is minimum is the matching point. In the figures above, false matching points of image captured by stereo vision are removed with object function and the epipolar line is recovered by fundamental matrix that is recalculated by least square method. According to the figure, errors exist in the recovered epipolar line and the quantity of recovered polar line is distinct in left and right image. So the accuracy of fundamental matrix is improved by optimization function.

\subsection{The epipolar line of corresponding feature po- ints recovered by fundamental with PSO al- gorithm}

Figures above show that the epipolar line of the figures of the character and rock which are captured by
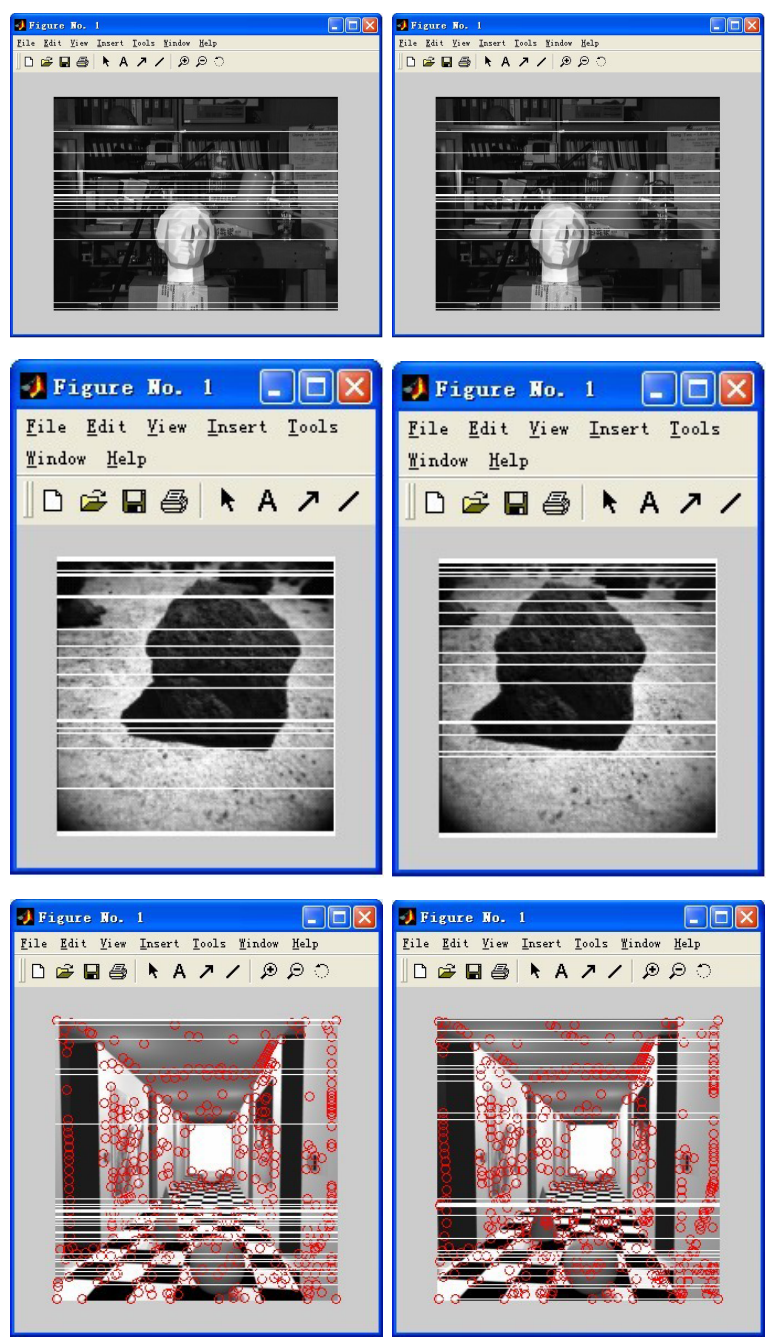

Figure 2 Restored polar line of feature points

camera is recovered. In which, we optimize the fundamental matrix with PSO algorithm. The result shows that the accuracy of epipolar line that is recovered is improved obviously and the quantity of the epipolar lines of the corresponding points are basically the same in the figures.

Like Table 1, the residual of various polar equations are compared. From the figure, the fundamental matrix is calculated with the feature points directly extracted from figures. But, a lot of false matching points, the error of polar line of matching feature points is large between left and right camera. Therefore, epipolar line is exactly restored by accurate fundamental matrix; according to the object function set by the known conditions, error matching points are eliminated, and the fundamental matrix is calculated by correct matching points. The results show that the polar line of feature points is better restored by the fundamental matrix which is optimized with PSO algorithm. 

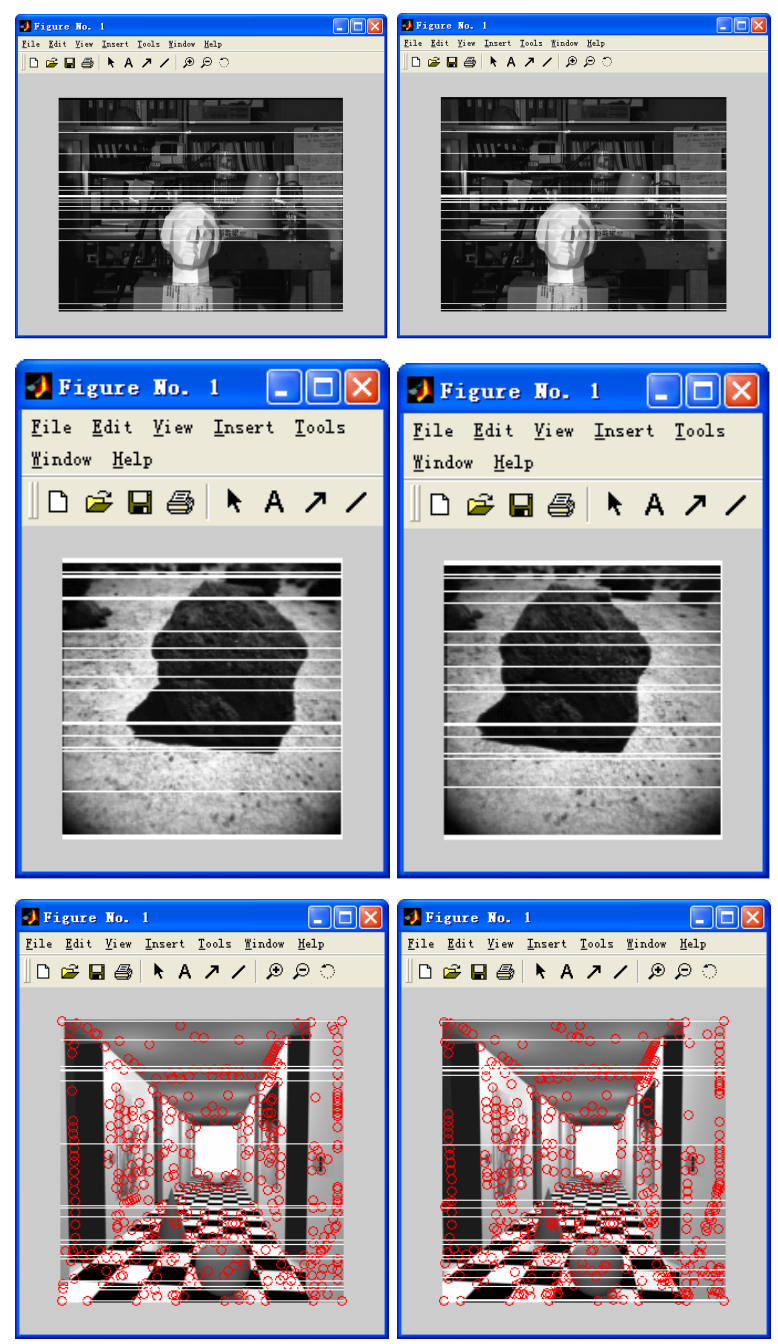

Figure 3 Restored polar line of feature points that false matching points are eliminated

Table 1 The residuals of polar equation of various methods

\begin{tabular}{|l|l|l|l|}
\hline & \multicolumn{3}{|c|}{$\begin{array}{c}\text { Polar equation residuals } \\
(\Delta, \text { Unit: Pixel) }\end{array}$} \\
\hline & $\begin{array}{l}\text { Character } \\
\text { figure }\end{array}$ & $\begin{array}{l}\text { Rock } \\
\text { figure }\end{array}$ & $\begin{array}{l}\text { Corridor } \\
\text { figure }\end{array}$ \\
\hline $\begin{array}{l}\text { Feature } \\
\text { extraction (the } \\
\text { (error matching } \\
\text { points are not } \\
\text { removed ) }\end{array}$ & 13.0988 & 13.1302 & 13.3902 \\
\hline $\begin{array}{l}\text { Least-squares } \\
\text { method } \\
\text { (excluding the } \\
\text { error matching } \\
\text { points) }\end{array}$ & 1.2512 & 1.3013 & 1.2035 \\
\hline PSO & & & \\
\hline
\end{tabular}

\section{Conclusion}

In this paper, Harris Operator is effectively used to extract feature points of various regions of the fig-
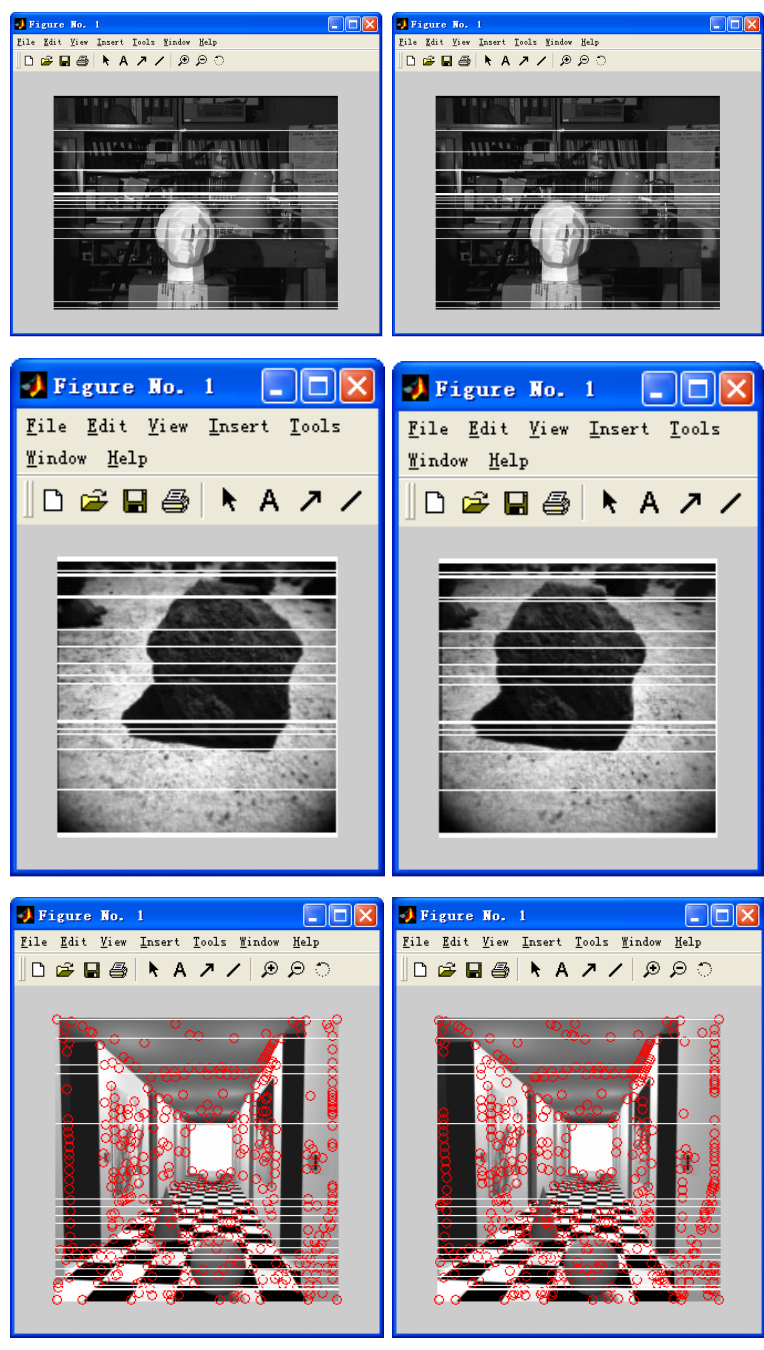

Figure 4 Restored polar line of feature points with fundamental matrix is optimized by PSO

ures. Then, the fundamental matrix is optimized by PSO algorithm after false matching points are eliminated with the object function which is founded by the theory of the distance between corresponding point and epipolar line. According to the experimental figures shown above, the fundamental matrix that is optimized with PSO algorithm has favorable effect on restored corresponding feature points polar line. Only Fundamental matrix is constraint relations that are gained from non-targeted sequence images. So, the important work that we will do next step is to research the fundamental matrix continuously.

\section{Acknowledgments}

This work is supported by Liaoning Province Educational Office Fund, China (No.20080611). 


\section{References}

[1] S. Brandt, Maximum Likelihood Robust Regression with Known and Unknown Residual Models, in ECCV 2002, 2002: 97-102.

[2] R.Hartley, In Defense of the Eight-Point Algorithm, IEEE Trans Pattern Analysis \& Machine Intelligence, 1997,19(6): 580-593

[3] Yi cheng tao, Wang xiao tong , Xu xiao gang, A Semiautomatic System of Image-based Modeling, Journal of Wuhan University of Technology (Transportation Science \& Engineering), 2009.33(2)

[4] Feng lin ,Zhang ming ju ,He ming feng ,Wang xiao dong, Teng hong fei , A Robust Point Matching Algorithm Based on Particle Swarm Optimization, Journal of System Simulation, 2004.8.

[5] Zhou dong xiang, Cai xuan ping ,Sun mao yin, A Eeature-Constrained Stereo Matching Algorithm, Journal of Image and Graphics, 2001.7.

[6] Wang Xu guang, Wang Zhi heng, Wu Fu chao. Harris Correlation and Feature Matching, Pattern Recognition and Artificial Intelligence, 2009,4

[7] Zheng You zhang. A Robust Technique For Matching Two Uncalibrated Images Through the Recovery of the Unknown Epipolar Geometry, Institut $\mathrm{Na}$ tional Derechercheen Inforrmatique eten Automatique,May, 1994.

[8] ZHANG Wen-ming, LIU Bin, LI Hai-bin. Characteristic point extracts and the match algorithm based on the binocular vision in three dimensional reconstruction. Optical Technique.2008.

[9] LIU Bo, HU Zheng-ping, WANG Cheng-ru. Multilevel interactive image enhancement algorithm based on fuzzy relaxation iterative procedure. Optical Technique.2009.

[10] Houya Li, Li Gang, Wang Wei, Improvement of Levenberg-marquardt Algorithm in Target Identification by Particle Swarm Optimizing Algorithm. Journal Of Detection \& Control .2010.

[11] Tang Xin-xing,Zhao Ding-xuan,Huang Haidong,Feng Shi-zhu. Modified stereo vision calibration method for construction robot. Journal Of JiLin University (Engineering and Technology Edition) .2007 . 